\title{
Esophageal Liposarcoma with Cervical and Abdominal Approach: A Case Report
}

\author{
Rubén A. Uvalle-Villagómez, David Pérez-Salazar, Raúl Martínez-Zarazua, Roberto Pineda-Quiñones, \\ Jaime Rodríguez-García and Marco A. Treviño-Lozano \\ Department of General Surgery, School of Medicine, “Dr. José Eleuterio González” University Hospital, Autonomous University of Nuevo Leon, NL, \\ Mexico
}

\begin{abstract}
Introduction: Liposarcoma is the most common tumor in soft tissues, being extremely rare in the esophagus. Its diagnostic approach is difficult due to its clinical presentation, being able to be confused with motor pathologies. The definitive treatment is surgical resection, which, together with anatomopathological analysis, is considered the only diagnostic methods. Case Report: A 59-year-old female, with a clinical picture of 5 years of evolution, retrosternal pain accompanied by gastroesophageal reflux, progressive dysphagia, weight loss, and dyspnea. She was assessed with endoscopy and tomography showing a polypoid pedunculated lesion in the esophagus, on which is performed surgical treatment with a resection, diagnosing liposarcoma. Conclusion: Liposarcoma is the most common tumor found in soft tissues, being a disease of very low incidence in the esophagus. It is a slow-growing neoplasm, with uncertain pathophysiology, reaching sufficient size late in its diagnostic approach, developing obstructive symptoms in the superior digestive tract, and in more severe cases, affecting the airway. It appears mostly in adults, and surgical resection and anatomopathological analysis are the definitive surgical treatments and methods of choice for diagnosis.
\end{abstract}

Key words: Esophageal liposarcoma. Esophageal cancer. Mesenchymal tumor.

\section{Introduction}

Liposarcoma is the most common tumor in soft tissues in adult age, representing $20 \%$ of mesenchymal malignancies, occurring most commonly in retroperitoneum, soft tissues of the lower limbs and chest, and is extremely rare in the esophagus, with an incidence of $0.5 \%{ }^{1}$. Originating from the esophageal mucous and submucous $^{2}$, they are polypoid. However, there have been reports of lesions with a transmural invasion ${ }^{3}$. Today, they are classified histologically into subtypes: dedifferentiated, well-differentiated, pleomorphic, or myxoid. The most common histological subtype is well-differentiated liposarcoma; the majority are polypoid $(75 \%)$ in comparison to being sessile (22\%). The origin in most cases is in the proximal portion of the esophagus. ${ }^{4}$ Its pathology is uncertain; a theory on the development of this disease is the presence of abnormal growth of the layers of the esophagus, especially in the submucous, taking the shape of a diverticulum, which with traction and peristalsis causes the insidious growth of the intramural tumor ${ }^{5}$. This is a slow-growth lump, which occurs in the esophagus without causing symptoms until it reaches a diameter big enough to impede or alter esophageal transit, or in worse cases, alter the respiratory cycle. Its symptomatology makes diagnosis difficult, as it mimics esophageal motor alterations. Patients can present progressive dysphagia, a feeling of oppression

\section{Correspondence:}

Rubén Alejandro Uvalle-Villagómez

E-mail: alejandro_1986@ hotmail.com
Available online: 18-01-2019

Date of reception: 16-10-2018

Date of acceptance: 27-11-2018

DOI: 10.24875/RMU. 18000033
Medicina Universitaria. 2018;20(4):172-174 www.medicinauniversitaria.org CC BY-NC-ND license (http://creativecommons.org/licenses/by-nc-nd/4.0/). 
around the neck, dyspnea, weight loss, nausea, and retrosternal pain, and in some cases, regurgitation of the lesion in the oral cavity can occur, causing occlusion of airway and death. According to the literature, this pathology has a higher prevalence in men, presenting dysphagia as their most common symptom. Weight loss occurs in $25 \%$ of patients, and in rare cases, gastrointestinal bleeding. Imaging studies, barium tests, manometries, and endoscopies are of limited use since they could confuse diagnosis. The most commonly used imaging study and the one which has been found to be the most useful is a tomography. The diagnostic method and definitive treatment consist of resection and anatomopathological study of the lesion. Invasive biopsy studies of the lesion have not been conclusive or useful for diagnosis; only an estimated $5 \%$ of cases have been diagnosed using these studies. Definitive treatment can be performed through different types of surgical approaches, either laparotomy, cervicotomy, or thoracotomy, and there are reports of a transoral resection of the lesion through an endoscopy in cases with small lesions ${ }^{6}$.

\section{Case report}

A 59-year-old female with a history of occasional drinking and smoking, with an inguinal hernioplasty at 49 approximately, three pregnancies, and three deliveries. The condition onsets after presenting a referred intermittent epigastric pain with approximately 3 years of evolution, accompanied by dyspepsia and gastroesophageal reflux, which had been treated with proton-pump inhibitors, which reduced symptomatology. The symptoms progress for a year without presenting changes with treatment and the patient refers to weight loss, progressive dysphagia from solids to liquids, dysphonia, and dyspnea in the past 3 months. She is assessed with an endoscopy showing a gigantic hiatal hernia and unaltered gastric mucous, in addition to an extrinsic compression of the esophagus, for which an etiology is not determined. Assessment continues with a tomography with contrast, where we are able to observe an intramural esophageal tumor, a polypoid pedunculated lesion in the esophagus, necrotic, and spreading to the gastroesophageal junction, with a length of approximately $20 \mathrm{~cm}$, without findings of lymphadenopathies or metastasis.

During the assessment, we find the patient to be malnourished, with a weight of $35 \mathrm{~kg}$, tachycardia, and polypnea. The patient is stabilized and given surgical management, performing a left lateral cervicotomy focused on the esophagus and identifying the origin of

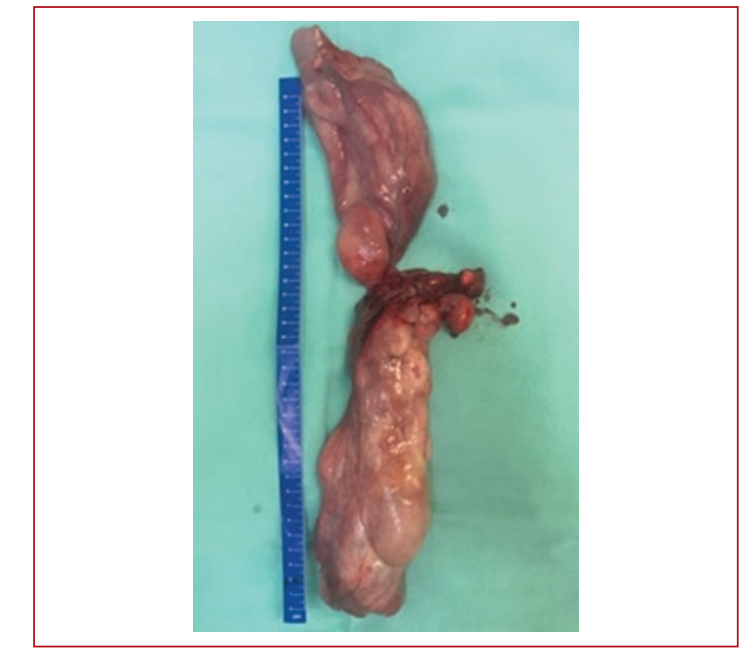

Figure 1. Product of lesion resection, which is observed to be polypoid and $>20 \mathrm{~cm}$.

the lesion. Resection of the pedicle is conducted; then, the abdomen is approached through supraumbilical laparotomy and gastrostomy, where we observe the distal portion of the lesion, which was free, thus resulting in extraction of the lesion through this incision without complications. A Nissen fundoplication is performed for hiatal hernia repair; a gastrostomy tube is placed as well as drainage on the surgical site for monitoring. The patient is placed in observation, presenting a positive immediate post-operative evolution, and is put on a gastrostomy diet. On her $3^{\text {rd }}$ post-operative day with the drainage on her neck reduced, removal of drainage is conducted, and the patient is discharged on the $4^{\text {th }}$ day of her hospital stay. A week after discharge, it was decided to begin an oral diet, and later, the gastrostomy tube was removed. The patient is currently in oncology monitoring, programmed with annual endoscopies, the latest of which was performed without any evidence of a relapse of the lesion. The anatomopathological study reports a well-differentiated liposarcoma, that is, $21 \mathrm{~cm}$ $\times 8 \mathrm{~cm} \times 3 \mathrm{~cm}$, with a weight of $590 \mathrm{~g}$ (Fig. 1), with a resection edge free of malignancies. Due to the morphology and location of the lesion, the immunohistochemical analysis was conducted for S-100, P16, and MDM2, finding them positive, confirming the diagnosis of liposarcoma.

\section{Discussion}

The presence of this pathology in the gastrointestinal tract is rare, and even more rare is its presence in the esophagus. Most tumors in the esophagus emerge 
from mucous, $<5 \%$ are of a mesenchymal origin, and only $0.5 \%$ are liposarcomas. There are few reports of cases with this pathology; the last report of a case that we are aware of is one by Rodriguez et al., in 2018, in Spain, with a patient with a well-differentiated liposarcoma, with the case number $43^{7}$.

In our case, in particular, we have a female patient (these lesions are predominant in men) treated with proton-pump inhibitors for abdominal pain, which resembled another pathology until swallowing alterations were added, with an unfinished endoscopy study, with the tomography being the study where it was possible to show the lesion where no metastasis was observed. Due to the characteristics and location of the lesion evidenced, it was decided to perform a cervicotomy to identify the base of the lesion and perform its resection, as well as an abdominal approach by laparotomy to remove the lesion and treat the hiatal hernia, obtaining a good evolution in the post-operative period and in its follow-up.

\section{Conclusion}

Liposarcoma is a very rare disease, where the symptoms appear in more advanced stages when the lesion has a sufficient diameter to produce them, resembling motor pathologies and without having a specific symptom that can help us in the early diagnosis. The prognostic factors of this disease are the histological grade, its location, and adequate resection in surgical treatment ${ }^{8}$. The degree of differentiation, as well as its complete microscopic resection (R0 resection), is the most important prognostic factors, which, in this case, were favorable. Usually, the surgical management of these includes thoracotomy, which, in this case, was changed to a laparotomy and removal of the lesion abdominally, given the large size of this lesion. Surveillance is recommended to identify recurrences through imaging studies.

The data of this study's subject have been protected, with the confidentiality of their data, their privacy, and informed consent obtained for the procedures performed during their evolution of the disease.

\section{Ethical disclosures}

Protection of human and animal subjects. The authors declare that no experiments were performed on humans or animals for this study.

Confidentiality of data. The authors declare that they have followed the protocols of their work center on the publication of patient data.

Right to privacy and informed consent. The authors declare that no patient data appear in this article.

\section{References}

1. Smith MA, Kluck E, Jagannath S, Yang SC. Giant multi-polypoid liposarcoma of the esophagus: an atypical presentation. Ann Thorac Surg. 2010; 89:610-2.

2. Liakakos T, Troupis T, Tzathas C, et al. Primary liposarcoma of esophagus: a case report. World J Gastrointest Oncol. 2016;8:835-9.

3. Sui X, Li Y, Zhao H, Wang J. Giant liposarcoma of the esophagus with li-fraumeni-like syndrome. Eur J Cardiothorac Surg. 2011;40:1253-5.

4. Dowli A, Mattar A, Mashimo $\mathrm{H}$, et al. A pedunculated giant esophageal liposarcoma: a case report and literature review. J Gastrointest Surg. 2014;18:2208-13.

5. Valiuddin HM, Barbetta A, Mungo B, Montgomery EA, Molena D. Esophageal liposarcoma: well-differentiated rhabdomyomatous type. World J Gastrointest Oncol. 2016;8:835-9.

6. Aloraini A, Nahal A, Ferri LE. Transoral endoscopic resection of esophageal liposarcoma. Ann Thorac Surg. 2012;94:e121-2.

7. Perez RA, Serrano PN, Tejero GA, Nalda EB, Albarellos GR. Liposarcoma gigante de esofago en paciente joven, asintomatico. Cir Esp. 2018;96:381-3.

8. Hasanabadi M, Amiri M, Tajedini A, et al. Huge myxoid liposarcoma of the esophagus: a case report. Acta Med Iran. 2011;49:118-21. 